\title{
RADIATIVE TRANSFER IN PRESTELLAR CORES: A MONTE CARLO APPROACH
}

\author{
D. Stamatellos \\ Department of Physics \& Astronomy, Cardiff University, Wales, UK \\ D.Stamatellos@astro.cf.ac.uk \\ A. P. Whitworth \\ Department of Physics \& Astronomy, Cardiff University, Wales, UK \\ A.Whitworth@astro.cf.ac.uk
}

\begin{abstract}
We use our Monte Carlo radiative transfer code to study non-embedded prestellar cores and cores that are embedded at the centre of a molecular cloud. Our study indicates that the temperature inside embedded cores is lower than in isolated non-embedded cores, and generally less than $12 \mathrm{~K}$, even when the cores are surrounded by an ambient cloud of small visual extinction $\left(A_{\mathrm{V}} \sim 5\right)$. Our study shows that the best wavelength region to observe embedded cores is between 400 and $500 \mu \mathrm{m}$, where the core is quite distinct from the background. We also predict that very sensitive observations $\left(\sim 1-3 \mathrm{MJy} \mathrm{sr}^{-1}\right)$ at $170-200 \mu \mathrm{m}$ can be used to estimate how deeply a core is embedded in its parent molecular cloud. Finally, we present preliminary results of asymmetric models of non-embedded cores.
\end{abstract}

\section{Introduction}

Prestellar cores are cores that are either on the verge of collapse or already collapsing (e.g. Myers \& Benson 1983, Ward-Thompson et al. 2002). They represent the initial stage of star formation and their study is important since theoretical models of star formation are very sensitive to the initial conditions.

Prestellar cores have been observed either isolated or embedded in protoclusters. Isolated prestellar cores (e.g. L1544, L43, L63) have extent $\gtrsim$ $1.5 \times 10^{4} \mathrm{AU}$ and masses $0.5-35 \mathrm{M}_{\odot}$ (Ward-Thompson et al. 1999, André et al. 2000). On the other hand, prestellar cores embedded in protoclusters (e.g. in $\rho$ Oph, NGC2068/2071) are generally smaller, with extent $\sim 2-4 \times 10^{3} \mathrm{AU}$ and masses $\sim 0.05-3 \mathrm{M}_{\odot}$ (Motte et al. 1998, Motte et al. 2001). 
In this paper, we present radiative transfer models of non-embedded and embedded prestellar cores, performed using a 3-D Monte Carlo radiative transfer code we have developed (PhAETHON).

\section{The Method: Monte Carlo Radiative Transfer}

Our method (Stamatellos \& Whitworth 2003) is similar to that developed by Wolf, Henning \& Stecklum (1999) and Bjorkman \& Wood (2001). We represent the radiation field of a source (star or background radiation) by a large number of monochromatic luminosity packets ( $L$-packets). These $L$-packets are injected into the system and interact stochastically with it. If an $L$-packet is absorbed its energy is added to the local cell and raises the local temperature. To ensure radiative equilibrium the $L$-packet is re-emitted immediately with a new frequency chosen from the difference between the local cell emissivity before and after the absorption of the packet (Bjorkman \& Wood 2001). This method conserves energy exactly, accounts for the diffuse radiation field and its 3 -dimensional nature makes it attractive for application in a variety of systems.

The code has been thoroughly tested using the thermodynamic equilibrium test (Stamatellos \& Whitworth 2003) and also against benchmark (Ivezic et al. 1997) and previous (Bjorkman \& Wood 2001) calculations.

\section{Non-Embedded Prestellar Cores}

We represent prestellar cores by Bonnor-Ebert (BE) spheres, in which gravity is balanced by gas pressure (Bonnor 1956, Ebert 1955). In many cases this is a good approximation to prestellar cores (e.g. Alves et al. 2001, WardThompson et al. 2002).

For the radiation incident on the core, we use the Black (1994) interstellar radiation field (hereafter BISRF), that consists of an optical component due to radiation from giant stars and dwarfs, a component due to thermal emission from dust grains, mid-infrared radiation from non-thermally heated grains and the cosmic background radiation.

The opacity of the dust at the low temperatures (5-20K) and high densities $\left(10^{4}-10^{7} \mathrm{~cm}^{-3}\right)$ expected in prestellar cores, is quite uncertain. In our study, we use the opacities calculated by Ossenkopf and Henning (1994), for grains that have coagulated and accreted thin ice mantles.

We find that the temperature inside non-embedded cores drops from around $17 \mathrm{~K}$, at the edge of the core, to a minimum at the centre, which maybe as low as $7 \mathrm{~K}$, depending on the visual extinction to the centre of the core. Our results are similar to those of previous studies (Evans et al. 2001, Zucconi et al. 2001). 


\section{Embedded Prestellar Cores}

The radiation field incident on cores embedded in molecular clouds is different from that incident on isolated non-embedded cores. The ambient molecular cloud absorbs the UV, optical and NIR part of the radiation and reemits it in the FIR and submm region (Mathis et al. 1983). It also makes the radiation incident on the core anisotropic, because, in general, the ambient cloud is not homogeneous. Another factor that contributes to the anisotropy of the radiation incident on an embedded core is the presence of stars or protostars in the vicinity of the core or the cloud (e.g as in $\rho$ Oph; Liseau et al. 1999).

The Model. In this first approach (Stamatellos \& Whitworth 2003), we study a spherical core at the centre of a molecular cloud of uniform density. The radiation incident on the molecular cloud is the BISRF, but the radiation incident on the core is enhanced in the FIR and submm and reduced at shorter wavelengths, as a result of the presence of the molecular cloud around the core.

We chose the parameters of our models so as to mimic the embedded prestellar cores and the conditions in the $\rho$ Oph protocluster (see Motte et al. 1998): core sizes $4-8 \times 10^{3} \mathrm{AU}$, masses $0.4-1.2 \mathrm{M}_{\odot}$, ambient cloud particle density $n_{\mathrm{tot}}=0.96 \times 10^{4} \mathrm{~cm}^{-3}$ and ambient cloud pressure $\sim 10^{6} \mathrm{~cm}^{-3} \mathrm{~K}$. In Figs. 1-2, we present our calculations for a supercritical core at gas temperature $T=15 \mathrm{~K}$ with mass $0.8 \mathrm{M}_{\odot}$, under external pressure $P_{\text {ext }}=10^{6} \mathrm{~cm}^{-3} \mathrm{~K}$, surrounded by a spherical ambient cloud with different visual optical depths.

Core Temperature Profiles. We find that the presence of even a moderately thick cloud $\left(A_{\mathrm{V}}=5\right)$ around the core, results in a less steep temperature profile inside the core than in the case of a core that is directly exposed to the BISRF. When there is no surrounding cloud (Fig. 1, dashed lines), the temperature drops from $\sim 16 \mathrm{~K}$ at the edge of the core to around $6-7 \mathrm{~K}$ in the centre ( $\Delta T \approx 9-10 \mathrm{~K}$, depending on the core density), whereas with a $\tau_{V}=5$ ambient cloud (Fig. 1, dotted lines) the temperature drops from around $11 \mathrm{~K}$ to $7 \mathrm{~K}$ $(\Delta T \approx 4 \mathrm{~K}$ ). Our studies show that dust temperatures inside embedded cores are probably lower than $12 \mathrm{~K}$ in cores surrounded by even a relatively thin cloud $\left(A_{\mathrm{V}} \approx 5\right)$, which seems to be the case for many of the prestellar cores in $\rho$ Ophiuchi. Previous studies (Motte et al. 1998, Johnstone et al. 2000) of cores in this region, assumed isothermal dust at temperatures from 12 to $20 \mathrm{~K}$, when calculating core masses from mm observations and, thus, they may have underestimated masses by a factor of 2 .

Recent studies (André et al. 2003, André, this volume) find similar temperature profiles, using a different approach, in which they estimate the effective radiation field incident on an embedded core from observations. 

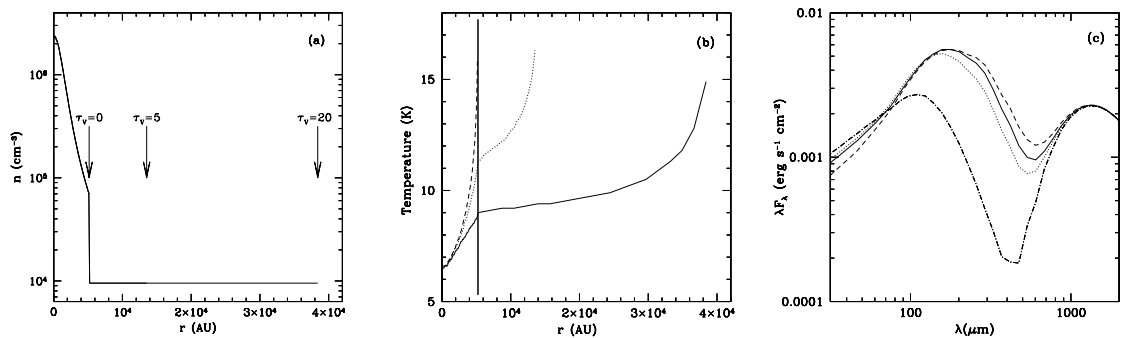

Figure 1. (a) Density, (b) dust temperature, and (c) SEDs, for a supercritical core (see text) surrounded by a spherical cloud with visual optical depth 20 (solid lines), 5 (dotted lines) and 0 (dashed lines). The dash-dot line on the SED graph corresponds to the background SED.

SEDs and Intensity Profiles. At 90 microns the core is seen in absorption against the background (Fig. 2a), and the intensity increases towards the edge of the core. For very centrally-condensed cores the decrease towards the centre is just $\sim 8-10 \mathrm{MJy} \mathrm{sr}^{-1}$, but for less centrally-condensed cores it is even lower. Thus, very sensitive (say $\sim 1-3 \mathrm{MJy} \mathrm{sr}^{-1}$ ) observations are needed to detect cores in absorption at $90 \mu \mathrm{m}$.
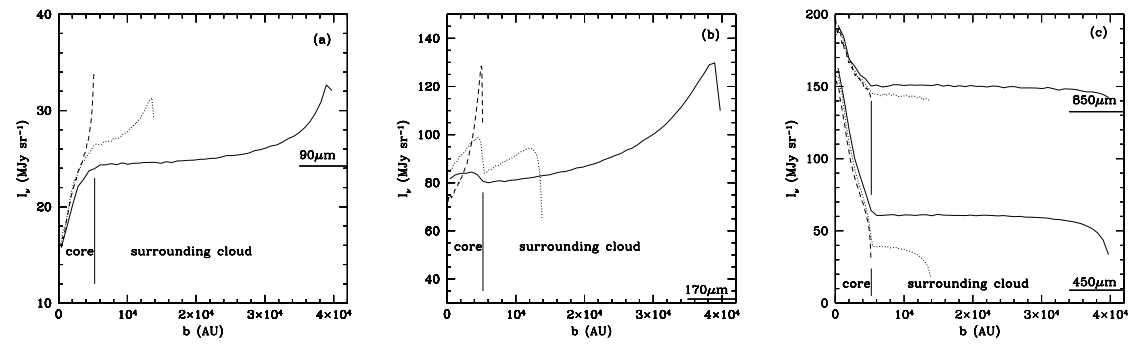

Figure 2. Intensity profiles at (a) 90 , (b) 170 , and (c) 450 and $850 \mu \mathrm{m}$, for the models in Fig. 1. The horizontal solid lines on the profiles correspond to the background intensity at the wavelength marked on the graph.

At wavelengths near the peak of the core emission $(150-250 \mu \mathrm{m})$ the intensity increases by a small amount $\left(\sim 5-20 \mathrm{MJy} \mathrm{sr}^{-1}\right.$ above the background $)$ towards the edge of the core (Fig. 2b). The higher the increase in the intensity near the core boundary, the less embedded is the core. Thus, very sensitive observations of embedded prestellar cores at 170-200 $\mu \mathrm{m}$, might allow us to determine the extinction of the cloud surrounding the core, and, thus, to estimate roughly the position of the core inside the cloud. However, more sophisticated modelling is required, with an accurate density profile for the cloud and taking into account the close environment of the core under study. 
Finally, at submillimeter and millimetre wavelengths $(400-1300 \mu \mathrm{m})$ the intensity drops toward the edge of the core considerably (Fig. 2c). The core can be easily observed at 400-500 $\mu \mathrm{m}$, where the contrast with the background is quite large $\left(\sim 50-150 \mathrm{MJy} \mathrm{sr}^{-1}\right)$. At wavelengths longer than $\sim 600 \mu \mathrm{m}$ the background radiation becomes important and the core emission is not much larger than the background emission ( 20-50 $\mathrm{MJy} \mathrm{sr}^{-1}$ larger).

Diagnostics. In Table 1, we list the peak intensities at various wavelengths for cores embedded in molecular clouds with visual optical depths 5 and 20. This table indicates that embedded cores are most easily distinguished from the background radiation around $450 \mu \mathrm{m}$. The peak emission

Table 1. Typical peak* intensities for embedded cores

\begin{tabular}{ccc}
\hline$\lambda(\mu \mathrm{m})$ & \multicolumn{2}{c}{$I_{\lambda}{ }^{\mathrm{a}}\left(\mathrm{MJy} \mathrm{sr}^{-1}\right)$} \\
& $\tau_{\text {cloud }}=5$ & $\tau_{\text {cloud }}=20$ \\
\hline \hline $90^{\mathrm{b}}$ & $5-15$ & $\sim 3$ \\
170 & $10-15$ & $\sim 3$ \\
450 & $55-160$ & $40-130$ \\
850 & $20-80$ & $15-70$ \\
1300 & $10-40$ & $10-25$ \\
\hline
\end{tabular}

* The term peak refers to the maximum intensity above or below the background (as noted) at a specific wavelength.

a Approximate peak intensities for a core embedded in a cloud with visual optical depth 5 and 20. The deeper the core is embedded the less distinct from the background is. The lower value corresponds to a subcritical core and the higher value to a supercritical (i.e. more centrally condensed) core.

${ }^{\mathrm{b}}$ At $90 \mu \mathrm{m}$ the core seen in absorption against the background.

from embedded cores could be as low as $\sim 10 \mathrm{MJy} \mathrm{sr}^{-1}$ above the background at $1300 \mu \mathrm{m}$, but it's at least $\sim 40 \mathrm{MJy} \mathrm{sr}^{-1}$ at $450 \mu \mathrm{m}$. The wavelength range between 400-500 seems favourable for observing embedded cores but the atmospheric transmission is not good in this range and space observations are needed. The upcoming Herschel (to be launched in 2007) will be operating in this range.

\section{Asymmetric Models of Non-Embedded Cores}

The model. Generally, cores are not spherically symmetric. Here, we assume non-embedded cores with a disk-like asymmetry, i.e. the core is denser on the equatorial plane;

$$
\rho(r, \theta)=\rho_{c} \frac{1+A\left(\frac{r}{r_{0}}\right)^{2} \sin (\theta)}{\left[1+\left(\frac{r}{r_{0}}\right)^{2}\right]^{2}},
$$


where $\rho_{c}$ is the the density at the centre of the core, $r_{0}$ the scale length, and $A$ is a factor that determines how asymmetric is the core. This density profile (Fig. 3, left) resembles the BE sphere density profile (drops as $r^{-2}$ at large radii and gets flatter near the centre). In Fig. 4, we present our calculations for a core with $n_{c}=10^{6} \mathrm{~cm}^{-3}, r_{0}=2 \times 10^{3} \mathrm{AU}, R_{\text {core }}=2 \times 10^{4} \mathrm{AU}, M=7.3 \mathrm{M}_{\odot}$ and $e=2.5$ ( $e$ is the ratio of the optical depth at $\theta=90$, i.e. looking at an edge-on core, to the optical depth at $\theta=0$ ).
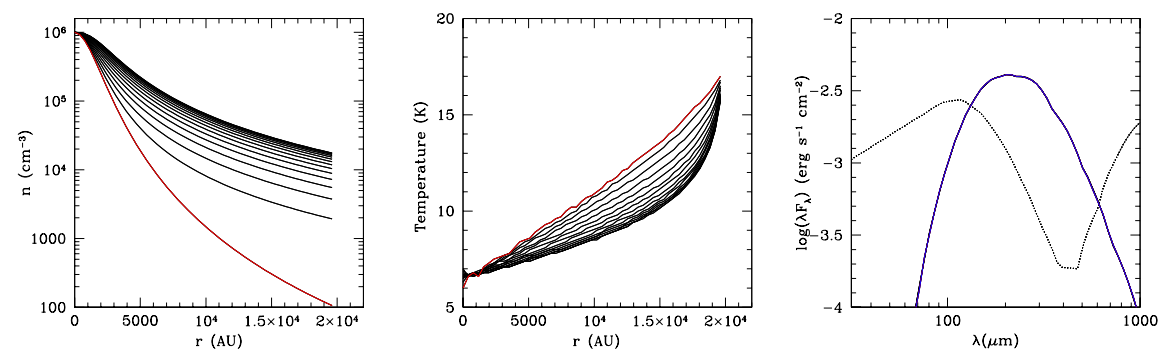

Figure 3. (a) Density, (b) dust temperature, and (c) SEDs, for an asymmetric core (see text). The core is divided into 15 equal-angle cells (each cell's angle span is $12^{\circ}$ ). The lower density cell corresponding to $\theta=0^{\circ}$ (lower curve on the density plot), has higher temperature (upper curve in the temperature plot). The dotted line on the SED graph corresponds to the incident SED.

Temperature Profiles. The dust temperature is $\theta$ dependent (Fig. 3, centre). As expected, the 'equator' of the core is colder than the 'poles'. The difference in temperature is around 3-4 $\mathrm{K}$ for the core under study $(e=2.5)$. The difference is expected to be larger for less symmetric cores.

SEDs and Images. The SED distribution (Fig. 3, right) of the core is the same at any viewing angle, because the core is optically thin to the radiation it emits (FIR and longer wavelengths). However, the isophotal maps are quite different for different viewing angles (Fig. 4).

At $200 \mu \mathrm{m}$ the outer, hotter parts of the core dominate the core emission. The core appears spherical when viewed pole-on and elongated when viewed edge-on. The image at $30^{\circ}$ is quite interesting, with two absorption 'blobs' near the centre of the core. A quick search through the Kirk (2003) sample of ISO/ISOPHOT observations did not reveal cores with such distinctive features, which is surprising, because one excepts some of the observed cores to be viewed at such angles. Further study is required to see whether this is due to the sensitivity and resolution of observations, due to selection effects, or it is connected with the core structure and its environment (i.e. the ambient cloud). 

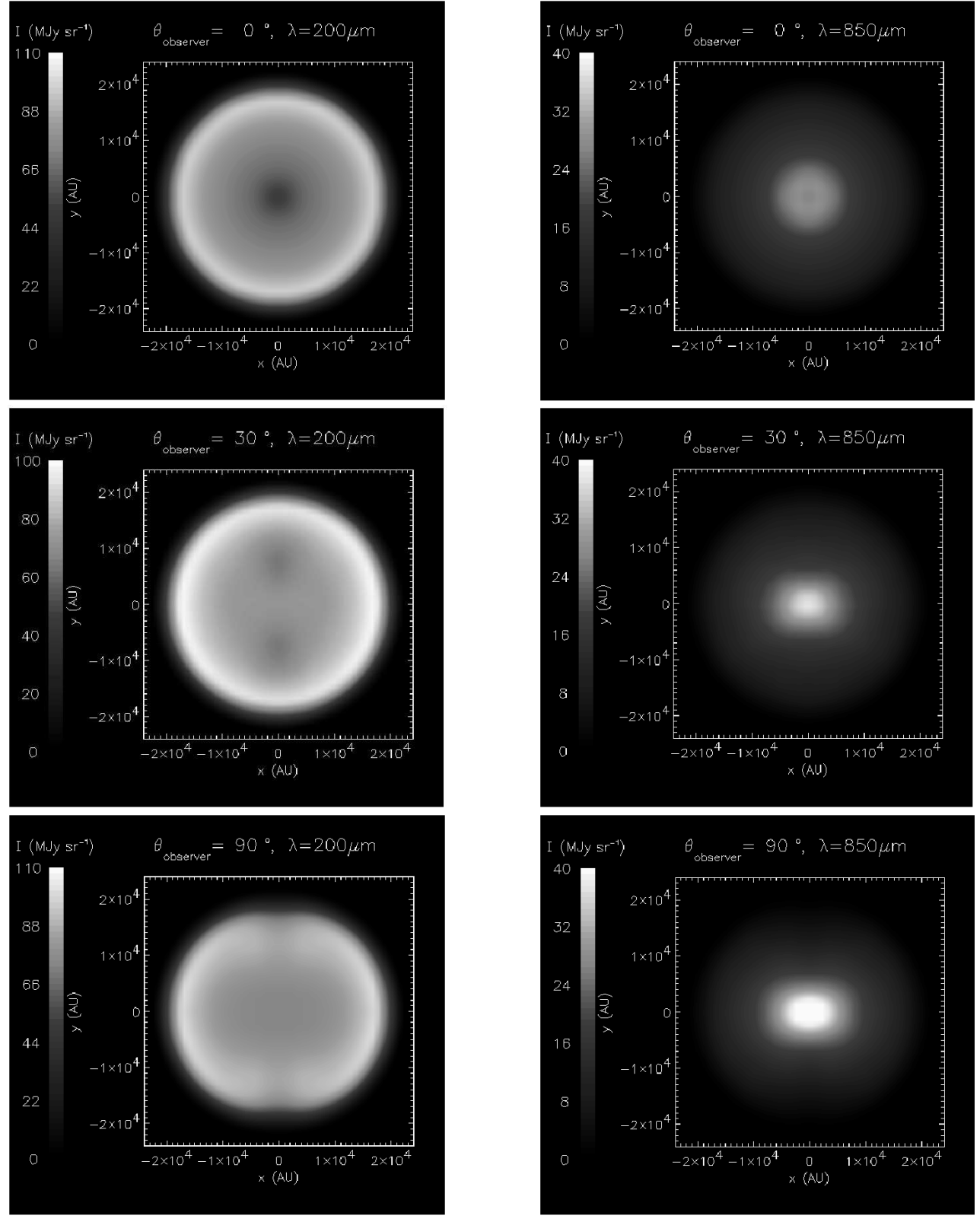

Figure 4. Isophotal maps at 200 (left panel) and $850 \mu \mathrm{m}$ (right panel) at viewing angles $0^{\circ}, 30^{\circ}$ and $90^{\circ}$ (top to bottom), for a disk-like asymmetric core (see text). The core appears elongated when viewed at a direction different from $\theta=0^{\circ}$. The colour image is available on the CD-ROM. 
At longer wavelengths, like $850 \mu \mathrm{m}$, the core emission is regulated by the column density. Thus, when the core is viewed edge-on the intensity is larger at the centre. For the same reason the core looks elongated when the observer is looking at it at any other direction than pole-on.

\section{Conclusions}

We applied a Monte Carlo radiative transfer method to spherical cores embedded inside molecular clouds. We find that the temperature inside these cores is less than $12 \mathrm{~K}$, even for an ambient cloud with moderate visual extinction $\sim 5$. We also studied asymmetric non-embedded cores and the preliminary results show that a small disk-like asymmetry in the density distribution will make the core look elongated when viewed at a random angle.

\section{Acknowledgments}

We acknowledge help from the EC Research Training Network "The Formation and Evolution of Young Stellar Clusters" (HPRN-CT-2000-00155).

\section{References}

André, P., Bouwman, J., Belloche, A., \& Hennebelle, P., 2003, astro-ph/0212492, to appear in the proceedings "Chemistry as a Diagnostic of Star Formation" (C.L. Curry \& M. Fich eds.)

André, P., Ward-Thompson, D., \& Barsony, M. 2000, Protostars and Planets IV, 59

Alves, J., Lada, C. J., \& Lada, E. A. 2001, Nature, 409, 159

Black, J. H. 1994, ASP Conf. Ser. 58: The First Symposium on the Infrared Cirrus and Diffuse Interstellar Clouds, 355

Bjorkman, J. E. \& Wood, K. 2001, ApJ, 554, 615

Bonnor, W. B. 1956, MNRAS, 116, 351

Ebert, R. 1955, Zeitschrift Astrophysics, 37, 217

Evans, N. J., Rawlings, J. M. C., Shirley, Y. L., \& Mundy, L. G. 2001, ApJ, 557, 193

Ivezic, Z., Groenewegen, M. A. T., Men'shchikov, A., \& Szczerba, R. 1997, MNRAS, 291, 121

Kirk, J., PhD Thesis, Cardiff, 2002

Johnstone, D., Wilson, C. D., Moriarty-Schieven, G., Joncas, G., Smith, G., Gregersen, E., \& Fich, M. 2000, ApJ, 545, 327

Liseau, R. et al. 1999, A\&A, 344, 342

Mathis, J. S., Mezger, P. G., \& Panagia, N. 1983, A\&A, 128, 212

Motte, F., Andre, P., \& Neri, R. 1998, A\&A, 336, 150

Motte, F., André, P., Ward-Thompson, D., \& Bontemps, S. 2001, A\&A, 372, L41

Myers, P. C. \& Benson, P. J. 1983, ApJ, 266, 309

Ossenkopf, V. \& Henning, T. 1994, A\&A, 291, 943

Stamatellos, D. \& Whitworth, A. P., 2003, submitted to A\&A

Ward-Thompson, D., Motte, F., \& Andre, P. 1999, MNRAS, 305, 143

Ward-Thompson, D., André, P., \& Kirk, J. M. 2002, MNRAS, 329, 257

Wolf, S., Henning, T., \& Stecklum, B. 1999, A\&A, 349, 839

Zucconi, A., Walmsley, C. M., \& Galli, D. 2001, A\&A, 376, 650 